\title{
Prevalence of Vitamin B12 deficiency in Type II diabetic patients using metformin.
}

1. FCPS (Medicine)

Assistant Professor Medicine

University of Lahore Teaching

Hospital, Lahore.

2. FCPS (Medicine)

Assistant Professor Medicine

Shalamar Hospital, Lahore.

3. MBBS, MRCP

Associate Professor Pulmonology,

Shalamar Hospital, Lahore.

Correspondence address:

Dr. Tahir Ullah Khan

Department of Medicine

University of Lahore Teaching Hospital,

Lahore.

tahirnurar69@gmail.com

Article received on:

09/07/2019

Accepted for publication:

$25 / 02 / 2020$

\begin{abstract}
Tahir Ullah Khan', Rozina Arshad ${ }^{2}$, Saleem Uz Zaman Adhami ${ }^{3}$
\end{abstract}
\begin{abstract}
Objectives: To determine the prevalence of Vitamin B12 deficiency in type II diabetic patients using metformin. Study Design: Cross Sectional study. Setting: Endocrinology Unit Shalamar Hospital Lahore. Period: July to September 2017. Material \& Methods: All of our patients were having previously diagnosed type II diabetes and using metformin for more than six months. Using strict exclusion criteria, vitamin B12 levels of patients were measured and analyzed. Results: It was evident from the present study that $27.33 \%$ of type II diabetic patients using metformin were having vitamin B12 levels less than $150 \mathrm{pg} / \mathrm{ml}$. Furthermore, our study showed that smokers are more liable to develop vitamin B12 deficiency than the nonsmokers. Also, use of multivitamins (containing vitamin B12) had a protective role against vitamin B12 deficiency. Conclusion: Long term use of metformin in type II diabetic patients is strongly associated with Vitamin B12 deficiency, therefore endocrinologists and physicians should take into consideration this significant adverse effect of metformin and screen for vitamin B12 deficiency in diabetic population before starting metformin therapy.
\end{abstract}

Key words: Metformin, Type II Diabetes Mellitus, Vitamin B12 Deficiency.

Article Citation: Khan T, Arshad R, Adhami Saleem Uz Zaman. Prevalence of Vitamin B12 deficiency in Type II diabetic patients using metformin. Professional Med $\mathrm{J}$ 2021; 28(9):1322-1325. https://doi.org/10.29309/TPMJ/2021.28.09.3926

\section{INTRODUCTION}

Diabetes mellitus is a common endocrine disorder characterized by persistent hyperglycemic state secondary to impaired metabolism of carbohydrates, proteins and fats. About 5 to $7 \%$ of US population is affected by diabetes and majority of them are having type II diabetes mellitus, mainly due to sedentary lifestyle and unhealthy eating practices. ${ }^{1}$

According to a data collected by Ramachandran and his team in 2012, more than 7.2 million Pakistanis are suffering from diabetes mellitus and its prevalence in urban population (10.6\%) is more than in rural $(7.7 \%)$ areas. ${ }^{2}$

Among all the oral hypoglycemic drugs used in type 2 diabetes mellitus, biguanides e.g. metformin are most commonly prescribed. For years, metformin has been known to cause gastrointestinal upset greatly limiting its efficacy and patient compliance, but the often neglected and least discussed side effect of this prototype dug is vitamin B12 deficiency, which can lead to subacute combined degeneration of spinal cord in some cases. ${ }^{3,4}$

The purpose of the study was to determine prevalence of vitamin B12 deficiency in type 2 diabetic patients on long term metformin therapy.

\section{MATERIAL \& METHODS}

This cross sectional study was conducted at endocrinology and diabetes unit Shalamar Hospital Lahore from $1^{\mathrm{ST}}$ July to $30^{\mathrm{TH}}$ September 2017. The study was approved by ethical committee (SMDC-IRB/AL/114/2021). Our Sample size was 150 with $95 \%$ confidence interval. Patients included in the study were those who had previously diagnosed type II diabetes, aged more than 35 years and using metformin for more than six months. Patients excluded from the study were those who had chronic renal failure as evident from persistently elevated serum creatinine $>2.0 \mathrm{mg} / \mathrm{dl}$, patients who previously had gastric bypass procedure, 
ileal resection or gastrectomy, those using other drugs causing vitamin B12 deficiency e.g. proton pump inhibitors, history of ethanol use, having prior history of autoimmune pernicious anemia or thyroid illness.

After obtaining a written informed consent, patients' data was collected as per our designed Performa which included patient name, age, sex, duration of diabetes, complete drug history including metformin (dosage and duration) and symptoms of peripheral neuropathy. Ensuring complete aseptic measures, patients' venous samples were obtained and using DXI automated analyzer, vitamin B12 level was measured. Patients having vitamin B12 level less than $150 \mathrm{pg} / \mathrm{ml}$ were labeled as vitamin B12 deficient while those having vitamin B12 levels more than $220 \mathrm{pg} / \mathrm{ml}$ were labeled as having adequate levels. All the data obtained was analyzed using SPSS version 23. Quantitative variables like age, duration of diabetes and metformin use, serum vitamin b12 level, were expressed as standard deviation, while qualitative variables e.g. gender were expressed as frequencies and percentages. $P$ value of 0.05 or less was chosen as statistically significant.

\section{RESULTS}

A total of 150 patients were enrolled in our study with age group of 35 to 75 years, mean age of the patients being $56.49 \pm 6.35$ years. Male gender was dominant (83 patients, 55.33\%) (Figure-1). Other baseline characteristics are shown in Table-l.

There was a wide range in serum vitamin B12 values, from 110 to $2100 \mathrm{pg} / \mathrm{ml}$. About 41 (27.33\%) patients had serum vitamin B12 level less than $150 \mathrm{pg} / \mathrm{ml}$ while $19(12.66 \%)$ patients had vitamin $\mathrm{B} 12$ level in intermediate range. The remaining $90(60.00 \%)$ patients had vitamin b12 level in the normal range i.e. more than 220pg/ $\mathrm{ml}$ (Figure-2). Regarding metformin dosage, 43 $(28.66 \%)$ were using $2500 \mathrm{mg} / 24 \mathrm{hr}$ or more, $9(6.00 \%)$ were using less than $850 \mathrm{mg} /$ day, and the remaining $98(65.33 \%)$ were using a dose between $850 \mathrm{mg}$ and $2500 \mathrm{mg}$ per day (Figure-3). Additional findings in our study were that smokers are more liable to vitamin b12 deficiency than nonsmokers $(P<0.001)$ and multivitamins have a protective role against vitamin B12 deficiency $(P<0.05)$. Of those who did not develop vitamin b12 deficiency, about 40 (26.66\%) were using multivitamins (containing Vitamin B12) and only 3 patients developed vitamin B12 deficiency (7.5\%). The study also showed that longer the duration of diabetes, more are the chances of vitamin b12 deficiency ( $p$ value 0.04). Additionally, longer the period of metformin use, more are chances of vitamin b12 deficiency ( $p$ value0.001). There was no significant impact of body mass index on Vitamin B12 in both groups.

\begin{tabular}{|l|c|}
\hline \multicolumn{1}{|c|}{ Variable } & Mean Value \\
\hline Age (Years) & $66.49 \pm 13.35$ \\
\hline $\begin{array}{l}\text { Duration of diabetes mellitus } \\
\text { (Mean, Years) }\end{array}$ & $9.16 \pm 5.59$ \\
\hline BMI (Mean, SD) & $29.43 \pm 5.01$ \\
\hline Smoking & $41(27.33 \%)$ \\
\hline B12 level (Mean, SD) & $432 \pm 240$ \\
\hline Metformin use & $150(100 \%)$ \\
\hline \begin{tabular}{l} 
Multivitamin use (containing vitamin B12) \\
Table-I. Baseline characteristics and mean Vitamin \\
\multicolumn{2}{|c}{ B12. }
\end{tabular} \\
\hline
\end{tabular}

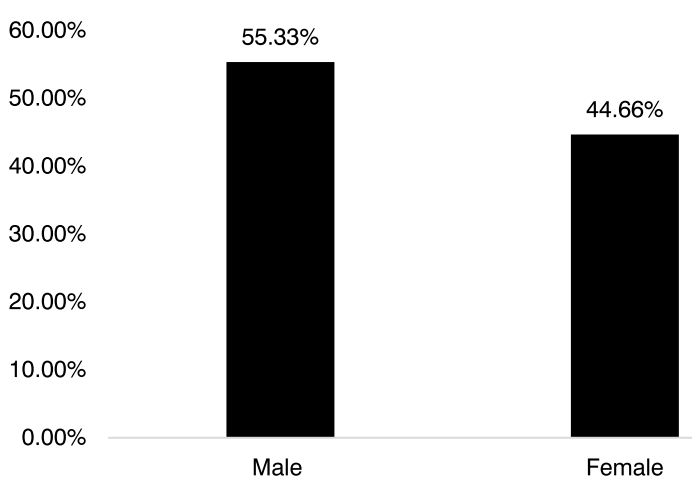

Figure-1. Gender distribution

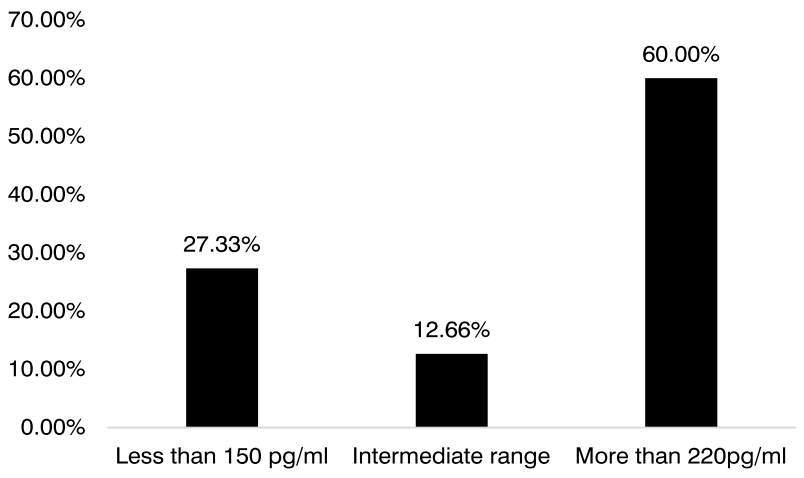

Figure-2. Vitamin B12 values. 


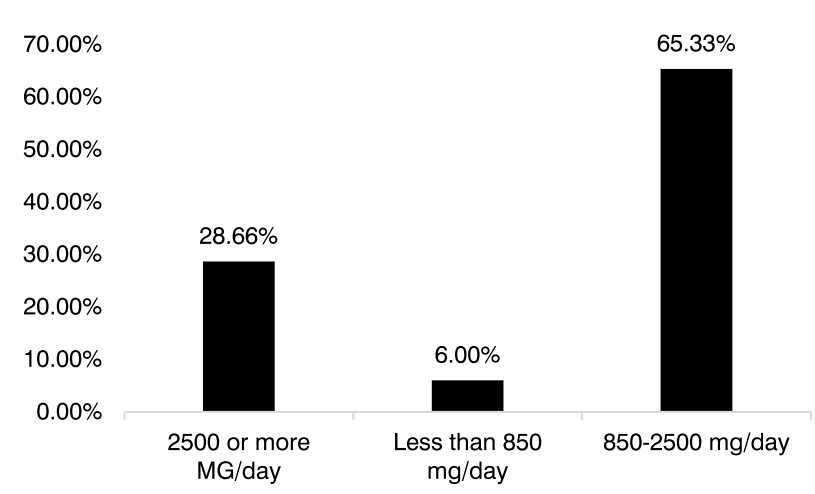

Figure-3. Different groups of Metformin dosage.

\section{DISCUSSION}

Metformin is the first line of all the oral hypoglycemic drugs prescribed in type 2 diabetics but the exact mechanisms responsible for vitamin B12 deficiency are poorly understood and need further exploration. ${ }^{3,4}$ Metformin was thought to be causing vitamin B12 deficiency by affecting intrinsic factor-vitamin B12 complex formation via its calcium dependent ileal cell membrane action, but more complex mechanisms may be involved and are yet to be discovered. ${ }^{5,6}$

In our study, 27.33\% diabetic patients using metformin for more than six months were having low $(<150 \mathrm{pg} / \mathrm{ml})$ vitamin B12 levels. Data available on this topic is limited one, but a few studies conducted so far have shown almost similar results with some variations. A study conducted at medicine department Combined Military Hospital Kharian in 2012 by Raheel Iftikhar and his team showed that $31 \%$ of patients using metformin had vitamin B12 deficiency as compared to controls (8.6\%). Dose of metformin was inversely proportional to vitamin B12 levels with statistically significant $p$ value less than $0.001 .^{7}$ Another study conducted by Adnan and his colleagues at endocrinology unit, Hayat Abad medical complex Peshawar in 2016 showed that prevalence of vitamin B12 deficiency in noninsulin dependent diabetics using metformin for more than 12 months was about $25.4 \%$ which was near to our results. ${ }^{8}$ In addition, they adopted a more comprehensive way by classifying vitamin B12 deficiency as mild, moderate and severe. A cross sectional study conducted by damiao CP and his colleagues in $2016^{9}$ pointed out that vitamin B12 deficiency was more prevalent in type 2 diabetics using metformin $(22.5 \%)$ than those not using metformin(7.4\%).The factors which interfered with vitamin B12 levels were proton pump inhibitors and $\mathrm{H} 2$ receptor antagonists. Chapman LE and his colleagues conducted a similar study at department of nutritional sciences, university of surrey UK, in 2016 which concluded that there is a strong association between vitamin B12 deficiency and long term use of metformin. ${ }^{10}$ A similar study was carried out in Brazil in 2010 showed that $43.7 \%$ of type 2 diabetic patients on long term metformin were vitamin B12 deficient. ${ }^{11}$ There were two major limitations to our study. First, it was conducted without measuring methylmalonic acid level in serum which could have allowed us to identify vitamin B12 deficient patients in their asymptomatic phase. ${ }^{12}$ Secondly, dietary predilections of our study population were not taken into consideration which could alter serum vitamin B12 level in the long run. ${ }^{13}$

\section{CONCLUSION}

This study led to the confirmation that long term use of metformin in non-insulin dependent diabetes patients had a strong link with vitamin B12 deficiency. Therefore, it is vital for endocrinologists and physicians to be not only aware of this strong documented association between metformin and vitamin B12 deficiency but also screen for vitamin B12 deficiency. It is also vital for endocrinologists to differentiate between diabetic neuropathy and peripheral neuropathy due to vitamin B12 deficiency. Moreover, use of multivitamins containing vitamin B 12 had a protective role against vitamin B12 deficiency.

Copyright $(25$ Feb, 2020.

\section{REFERENCES}

1. Harris MI. Diabetes in America: Epidemiology and scope of the problem. Diabetes care. 1998; 21(Supplement 3):C11-C4.

2. Ramachandran A, Snehalatha C, Shetty AS, Nanditha A. Trends in prevalence of diabetes in Asian countries. World journal of diabetes. 2012; 3(6):110.

3. Viollet B, Guigas B, Garcia NS, Leclerc J, Foretz M, Andreelli F. Cellular and molecular mechanisms of metformin: An overview. Clinical science. 2012; 122(6):253-70. 
4. Nathan DM, Buse JB, Davidson MB, Ferrannini E, Holman RR, Sherwin R, et al. Medical management of hyperglycemia in type 2 diabetes: A consensus algorithm for the initiation and adjustment of therapy. Diabetes care. 2009; 32(1):193-203.

5. Bauman WA, Shaw S, Jayatilleke E, Spungen AM, Herbert V. Increased intake of calcium reverses vitamin B12 malabsorption induced by metformin. Diabetes care. 2000; 23(9):1227-31.

6. Tomkin G, Hadden D, Weaver J, Montgomery D. Vitamin-B12 status of patients on long-term metformin therapy. Br Med J. 1971; 2(5763):685-7.

7. Iftikhar R, Qadir A, Iqbal Z, Usman H. Prevalence of vitamin B12 deficiency in patients of type 2 diabetes mellitus on metformin: a case control study from Pakistan. Pan African Medical Journal. 2014; 16(1).

8. Khan A, Shafiq I, Shah MH. Prevalence of Vitamin B12 deficiency in patients with Type II diabetes mellitus on metformin: A study from Khyber Pakhtunkhwa. Cureus. 2017; 9(8).

9. Damiao CP, Rodrigues AO, Pinheiro MF, Cruz RAF, Cardoso GP, Taboada GF, et al. Prevalence of vitamin B12 deficiency in type 2 diabetic patients using metformin: A cross-sectional study. Sao Paulo Med J. 2016; 134(6):473-9. Epub 2017/01/12. doi: 10.1590/1516-3180.2015.01382111. PubMed PMID: 28076635.
10. Chapman LE, Darling AL, Brown JE. Association between metformin and vitamin B12 deficiency in patients with type 2 diabetes: A systematic review and meta-analysis. Diabetes Metab. 2016; 42(5):31627. Epub 2016/05/01. doi: 10.1016/j.diabet.2016.03.008. PubMed PMID: 27130885.

11. Nervo M, Lubini A, Raimundo F, Faulhaber GAM, Leite C, Fischer LM, et al. Vitamin B12 in metformintreated diabetic patients: A cross-sectional study in Brazil. Revista Da Associacao Medica Brasileira. 2011; 57(1):46-9.

12. Wolters M, Hermann S, Hahn A. Effect of multivitamin supplementation on the homocysteine and methylmalonic acid blood concentrations in women over the age of 60 years. European journal of nutrition. 2005; 44(3):183-92.

13. Rizzo G, Lagana AS, Rapisarda AM, La Ferrera GM, Buscema M, Rossetti $P$, et al. Vitamin B12 among vegetarians: Status, assessment and supplementation. Nutrients. 2016; 8(12). Epub 2016/12/06. doi: 10.3390/nu8120767. PubMed PMID: 27916823; PubMed Central PMCID: PMC5188422.

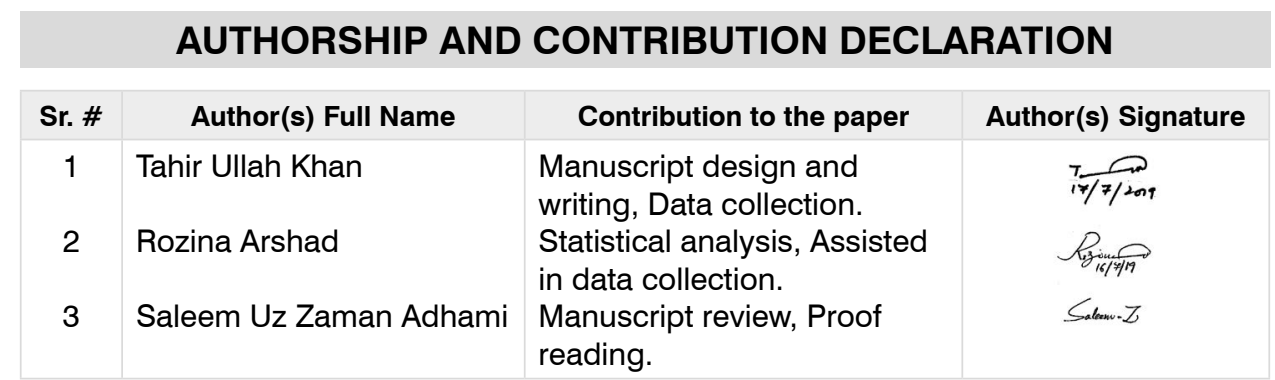

